\title{
Lifting Gomory Cuts With Bounded Variables
}

\author{
Gérard Cornuéjols* \\ Tepper School of Business, Carnegie Mellon University \\ Tamás Kis ${ }^{\dagger}$ \\ Computer and Automation Research Institute, \\ Hungarian Academy of Sciences \\ Marco Molinaro $\ddagger$ \\ Tepper School of Business, Carnegie Mellon University
}

December 2011, revised November 1012

\begin{abstract}
Recently, Balas and Qualizza introduced a new cut for mixed 0,1 programs, called lopsided cut. Here we present a family of cuts that comprises the Gomory mixed integer cut at one extreme and the lopsided cut at the other.
\end{abstract}

Keywords: Gomory mixed integer cut, split cut, lopsided cut, wedge inequality, Gomory function, lifting

\section{Introduction}

Recently, Balas and Qualizza [2] introduced a new cut for mixed 0,1 programs, called lopsided cut. Their derivation is based on the Balas-Jeroslow modularization technique [1]. Here we provide a geometric derivation of the lopsided cut and we generalize it to an infinite family. Our approach is to start from the classical Gomory function for continuous variables and to lift the coefficient of the integer variables using one of the bounds (say the upper bound of 1) following the technique introduced in [4]. It is convenient to present our geometric derivation using the infinite model first introduced by Gomory and Johnson [5]. We show that every inequality in our family is extreme for the corresponding infinite relaxation. We also show that our inequalities are split cuts. Finally we provide computational results.

\section{Setup}

We consider a linear equation where a bounded integer variable $x$ is expressed in terms of nonnegative variables. By a change of variable, we may assume that $x \leq 1$. Consider the upper

\footnotetext{
${ }^{*}$ Corresponding author: Gérard Cornuéjols, Tepper School of Business, Carnegie Mellon University, 5000 Forbes Av, Pittsburgh PA 15213, USA. Email: gc0v@andrew.cmu.edu. Supported by NSF grant CMMI1024554 and ONR grant N00014-09-1-0033.

${ }^{\dagger}$ Email: tamas.kis@sztaki.hu Supported by the János Bolyai research grant BO/00412/12/3.

${ }^{\ddagger}$ Email: molinaro@cmu.edu. Supported by NSF grant CMMI1024554, ONR grant N00014-09-1-0033, and an IBM PhD fellowship.
} 
bounded system

$$
\begin{gathered}
x=f+\sum_{r \in \mathbb{R}} r s_{r}+\sum_{r \in \mathbb{R}} r y_{r} \\
x \in\{-\infty, \ldots, 0,1\} \\
s_{r} \geq 0 \\
y_{r} \in \mathbb{Z}_{+}
\end{gathered}
$$

$(s, y)$ has finite support.

For simplicity of exposition, we focus on the case $0<f<1$. Note however that the approach that we present below can also be used when $f$ is further from the bound. We will fix $f \in(0,1)$ from now on. We use $I P$ to denote the set of $(x, s, y)$ feasible solutions for the above. We say that $(\psi, \pi)$ is a valid function if $\psi: \mathbb{R} \rightarrow \mathbb{R}$ and $\pi: \mathbb{R} \rightarrow \mathbb{R}$ are such that the inequality $\sum_{r} \psi(r) s_{r}+\sum_{r} \pi(r) y_{r} \geq 1$ is satisfied by all $(x, s, y) \in I P$.

Let $\psi_{G M I}$ denote the classical Gomory function for the coefficients of the continuous variables

$$
\psi_{G M I}(r)=\left\{\begin{array}{cc}
-\frac{r}{f} & r<0 \\
\frac{r}{1-f} & r \geq 0 .
\end{array}\right.
$$

Our goal is to lift $\psi_{G M I}$ for the integral variables, namely find $\pi$ such that

$$
\sum_{r} \psi_{G M I}(r) s_{r}+\sum_{r} \pi(r) y_{r} \geq 1
$$

is satisfied by all $(x, s, y) \in I P$, i.e., $\left(\psi_{G M I}, \pi\right)$ is a valid function.

Since integrality constraints on basic variables (i.e. on the left-hand side of the equation in $I P)$ are more easily handled, the idea is to 'transfer' the integrality of $y$ to a basic variable. For that, we first consider the extended system

$$
\begin{gathered}
\left(\begin{array}{l}
x \\
z
\end{array}\right)=\left(\begin{array}{l}
f \\
0
\end{array}\right)+\sum_{r \in \mathbb{R}}\left(\begin{array}{l}
r \\
0
\end{array}\right) s_{r}+\sum_{r \in \mathbb{R}}\left(\begin{array}{c}
r \\
\ell(r)
\end{array}\right) y_{r} \\
x \in\{-\infty, \ldots, 0,1\} \\
z \in \mathbb{Z} \\
s_{r} \geq 0 \\
y_{r} \in \mathbb{Z}_{+}
\end{gathered}
$$

We use $I P(\ell)$ to denote the set of feasible solutions $(x, z, s, y)$ for the above and observe that, when $\ell(r)$ is integral for all $r \in \mathbb{R}$, this extended system is equivalent to the original one.

Proposition 1. IP $=\operatorname{Proj}_{x, s, y} I P(\ell)$ for all $\ell: \mathbb{R} \rightarrow \mathbb{Z}$.

We assume $\ell: \mathbb{R} \rightarrow \mathbb{Z}$ in the remainder of the paper. Now we relax the integrality of the non-basics to obtain the system that we work with:

$$
\begin{gathered}
\left(\begin{array}{l}
x \\
z
\end{array}\right)=\left(\begin{array}{l}
f \\
0
\end{array}\right)+\sum_{r \in \mathbb{R}}\left(\begin{array}{l}
r \\
0
\end{array}\right) s_{r}+\sum_{r \in \mathbb{R}}\left(\begin{array}{c}
r \\
\ell(r)
\end{array}\right) y_{r} \\
x \in\{-\infty, \ldots, 0,1\} \\
z \in \mathbb{Z} \\
s_{r} \geq 0 \\
y_{r} \geq 0
\end{gathered}
$$

$(s, y)$ has finite support. 
Notice that this system partially captures the original integrality of $y$ and hence (its projection onto the $(x, s, y)$-space) is tighter than the relaxation obtained by completely dropping the integrality of $y$ from $I P$.

\section{$3 \quad$ Wedge inequalities}

We now construct the family of cuts which are the object of study in this paper. Let $S=$ $\{-\infty, \ldots, 0,1\} \times \mathbb{Z}$ and notice that every feasible solution $(x, z, s, y)$ for $Z(\ell)$ satisfies $(x, z) \in S$. For $\alpha \in(0,1]$, we consider the $S$-free convex set

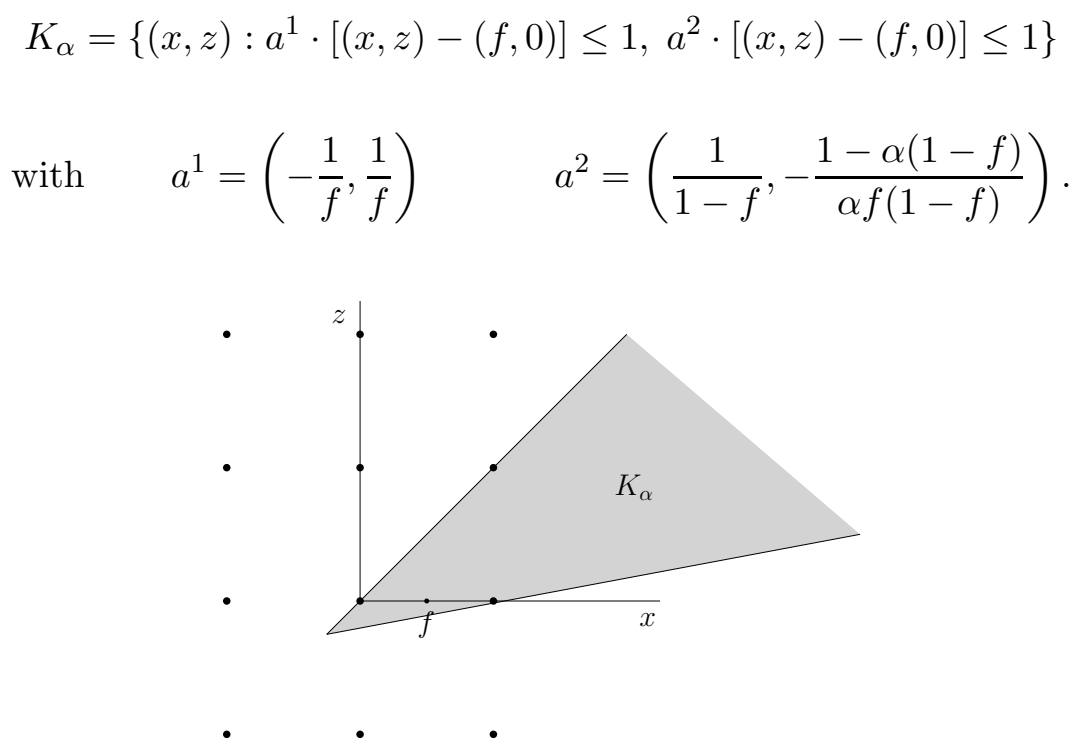

Figure 1: $S$-free convex set $K_{\alpha}$. The slope of the lower facet of $K_{\alpha}$ is $\frac{\alpha f}{1-\alpha(1-f)}$.

The $S$-free convex set $K_{\alpha}$ contains $(f, 0)$ in its interior and therefore it can be used to derive an intersection cut for $Z(\ell)$. More specifically, from the theory of $S$-free cuts [3], we obtain the valid inequality

$$
\sum_{r} \bar{\psi}(r) s_{r}+\sum_{r} \bar{\pi}_{\alpha}^{\ell}(r) y_{r} \geq 1
$$

with $\bar{\psi}(r)=\max \left\{a^{1} \cdot(r, 0), a^{2} \cdot(r, 0)\right\}$ and $\bar{\pi}_{\alpha}^{\ell}(r)=\max \left\{a^{1} \cdot(r, \ell(r)), a^{2} \cdot(r, \ell(r))\right\}$. We have the following explicit formula for the coefficients (see Figure 2):

$$
\begin{gathered}
\bar{\psi}(r)=\left\{\begin{array}{cc}
-\frac{r}{f} & r<0 \\
\frac{r}{1-f} & r \geq 0
\end{array}\right. \\
\bar{\pi}_{\alpha}^{\ell}(r)= \begin{cases}\frac{-r+\ell(r)}{f} & \ell(r)>\alpha r \\
\frac{r}{1-f}-\frac{\ell(r)(1-\alpha(1-f))}{\alpha f(1-f)} & \ell(r) \leq \alpha r .\end{cases}
\end{gathered}
$$

Inequality (1) will be called wedge inequality in the remainder. Since $\bar{\psi}=\psi_{G M I}$, the function $\left(\bar{\psi}, \bar{\pi}_{\alpha}^{\ell}\right)$ is a lifting of the Gomory function $\psi_{G M I}$. Geometrically this is clear: the Gomory function $\psi_{G M I}$ is obtained by considering the lattice-free set $[0,1]$ for the $x$-variable in $I P$. For all $\alpha$ the function $\bar{\psi}$ is obtained from the set $K_{\alpha}$ along rays $(r, 0)$, and therefore the relevant part of $K_{\alpha}$ for the function $\bar{\psi}$ is $K_{\alpha} \cap\{z=0\}$ which is the segment $[0,1] \times\{0\}$.

We remark that if $x$ is a 0,1 variable, another cut, exploiting the lower bound of 0 , can be obtained by substituting $x$ by $1-x$. 


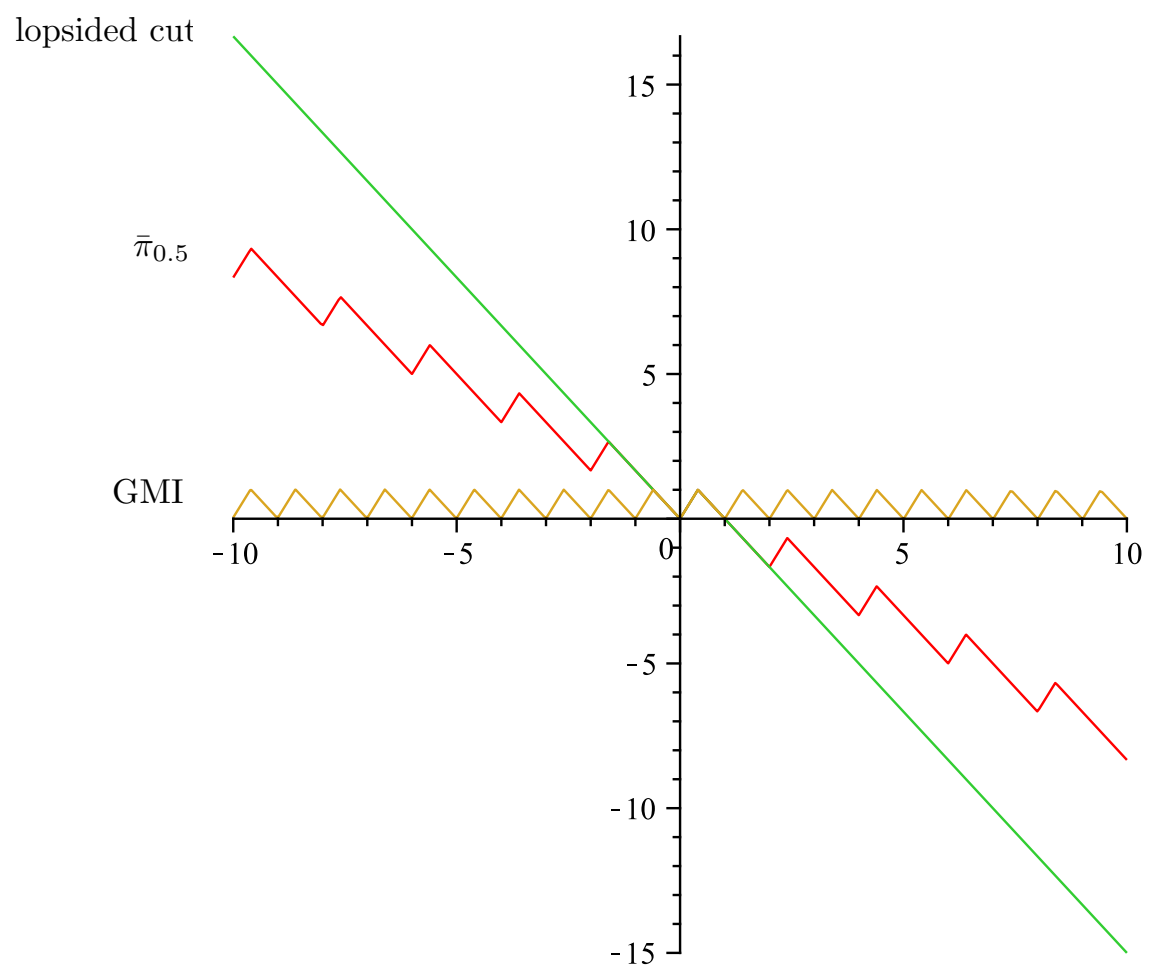

Figure 2: Graph of $\bar{\pi}_{0.5}$ and of functions corresponding to lopsided and GMI cuts.

\subsection{Optimizing $\ell$}

Notice that the cut above is valid for every $\ell: \mathbb{R} \rightarrow \mathbb{Z}$; we can choose the $\ell$ that gives the 'best' coefficients, i.e. the smallest value of $\bar{\pi}_{\alpha}^{\ell}(r)$. Thankfully, each ray is associated to a different component of $\ell$, so we can actually get the best coefficient for all the rays simultaneously.

Proposition 2. For given $r$ and $\alpha$, the value of $\ell$ that minimizes $\bar{\pi}_{\alpha}^{\ell}(r)$ is $\ell_{\alpha}(r)=\lceil\alpha(r+f-1)\rceil$. Furthermore $\bar{\pi}_{\alpha}^{\ell}(r)=\min \left\{\frac{-r+\lceil\alpha r\rceil}{f}, \frac{r}{1-f}-\frac{\lfloor\alpha r\rfloor(1-\alpha(1-f))}{\alpha f(1-f)}\right\}$.

Proof. Fix $r \in \mathbb{R}$. Note that $\bar{\pi}_{\alpha}^{\ell}(r)$ as a function of $\ell(r)$ is a piecewise linear function which is decreasing in the interval $(-\infty, \alpha r]$ and increasing in the interval $[\alpha r, \infty)$, hence with minimum at $\ell(r)=\alpha r$. Therefore, the minimum over all integer values of $\ell(r)$ is attained at either $\ell(r)=\lfloor\alpha r\rfloor$ or $\lceil\alpha r\rceil$. This shows the second part of the proposition.

To prove the first part, let $\tilde{\ell}$ be the (unique) value $\tilde{\ell} \leq \alpha r<\tilde{\ell}+1$ such that $\bar{\pi}_{\alpha}^{\tilde{\ell}}(r)=\bar{\pi}_{\alpha_{\sim}}^{\tilde{\ell}+1}(r)$. A simple calculation gives $\tilde{\ell}=\alpha(r+f-1)$. Since there is only one integer in the range $[\tilde{\ell}, \tilde{\ell}+1)$, the optimum choice of the integer $\ell(r)$ is $\ell_{\alpha}(r)=\lceil\alpha(r+f-1)\rceil$.

This has the following geometric interpretation: The optimum choice of $\ell$ is such that the ray $(r, \ell(r))$ belongs to the strip $(f, 0)+R$ (see Figure 3). This is related to the region of best possible liftings for wedges as introduced in [4] Section 3.1.

To simplify the notation, let $\bar{\pi}_{\alpha}=\bar{\pi}_{\alpha}^{\ell_{\alpha}}$.

\subsection{Limit cases}

Now we consider the extreme cases $\alpha=1$ and $\alpha \rightarrow 0$.

Proposition 3. For $\alpha=1, \bar{\pi}_{1}(r)=\min \left\{\frac{\lceil r\rceil-r}{f}, \frac{r-\lfloor r\rfloor}{1-f}\right\}$, i.e. we get the GMI cut. 


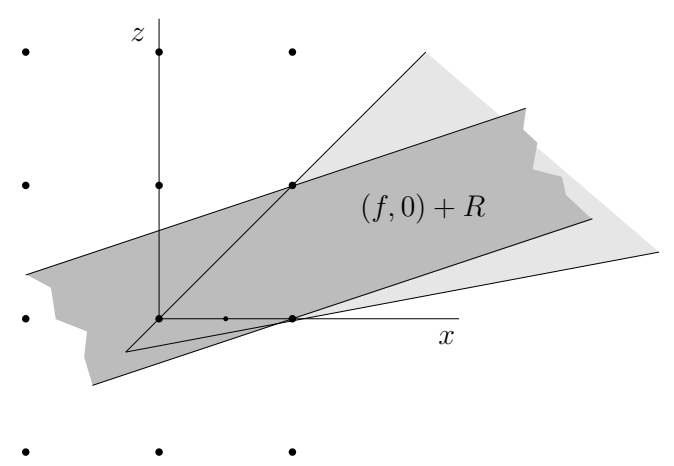

Figure 3: Region $(f, 0)+R$ of best possible liftings.

Now we focus on the case $\alpha \rightarrow 0$. Let

$$
\bar{\pi}_{0}(r)= \begin{cases}\frac{-r}{f} & r<0 \\ \frac{r}{1-f} & r \in[0,1-f] \\ \frac{-r+1}{f} & r>1-f .\end{cases}
$$

Lemma 1. Fix $r \in \mathbb{R}$. Then there exists $\alpha_{0}>0$ such that for any $0<\alpha \leq \alpha_{0}$, we have $\ell_{\alpha}(r)=0$ if $r \leq 1-f$ and $\ell_{\alpha}(r)=1$ if $r>1-f$. Hence, for all $0<\alpha \leq \alpha_{0}$ we have $\bar{\pi}_{\alpha}(r)=\bar{\pi}_{0}(r)$.

The above lemma directly gives the behavior of $\bar{\pi}_{\alpha}$ with $\alpha \rightarrow 0$.

Proposition 4. The pointwise limit $\lim _{\alpha \rightarrow 0} \bar{\pi}_{\alpha}$ equals $\bar{\pi}_{0}$, i.e. we get the lopsided cut of Balas and Qualizza [2].

\section{Strength of $\left(\bar{\psi}, \bar{\pi}_{\alpha}\right)$}

The main goal of this section is to show that the function $\left(\bar{\psi}, \bar{\pi}_{\alpha}\right)$ is extreme for $I P$. In fact we will prove an even stronger result, namely that this function is extreme in the 0,1 case. That is, we consider the more restricted system

$$
\begin{gathered}
x=f+\sum_{r \in \mathbb{R}} r s_{r}+\sum_{r \in \mathbb{R}} r y_{r} \\
x \in\{0,1\} \\
s_{r} \geq 0 \\
y_{r} \in \mathbb{Z}_{+}
\end{gathered}
$$

$(s, y)$ has finite support.

Since $I P$ is a relaxation of $\mathrm{B}(\mathrm{f}),\left(\bar{\psi}, \bar{\pi}_{\alpha}\right)$ is valid for the latter for all $\alpha \in[0,1]$.

We say that a valid function $(\psi, \pi)$ is extreme for $\mathrm{B}(\mathrm{f})$ if there are no distinct valid functions $\left(\psi_{1}, \pi_{1}\right)$ and $\left(\psi_{2}, \pi_{2}\right)$ such that $\psi=\frac{\psi_{1}}{2}+\frac{\psi_{2}}{2}$ and $\pi=\frac{\pi_{1}}{2}+\frac{\pi_{2}}{2}$. The following theorem formally states the main result of this section.

Theorem 1. For all $\alpha \in[0,1]$, the function $\left(\bar{\psi}, \bar{\pi}_{\alpha}\right)$ is extreme for $B(f)$.

In particular, this implies that we cannot improve the coefficients of the valid inequality for $I P$

$$
\sum_{r} \bar{\psi}(r) s_{r}+\sum_{r} \bar{\pi}_{\alpha}(r) y_{r} \geq 1
$$


even if we use the additional information that $x \in\{0,1\}$. We start by showing something weaker, namely that this inequality is minimal.

\subsection{Minimality}

We say that a valid function $(\psi, \pi)$ for $\mathrm{B}(\mathrm{f})$ is minimal if there is no other valid function $\left(\psi^{\prime}, \pi^{\prime}\right)$ such that $\psi^{\prime} \leq \psi$ and $\pi^{\prime} \leq \pi$. The following lower bound on valid functions is the main observation to prove that $\left(\bar{\psi}, \bar{\pi}_{\alpha}\right)$ is minimal.

Lemma 2. Let $(\psi, \pi)$ be a valid function for $B(f)$. Then:

1. $\psi \geq \bar{\psi}$

2. $\pi(r)+\pi(1-f-r) \geq 1$ for all $r \in \mathbb{R}$.

Proof. Consider the first property. Notice that for $r>0$, setting $\bar{s}_{r}=\frac{1-f}{r}$ and all other $\bar{s}_{r^{\prime}}$ 's equal to 0 gives a feasible solution for $\mathrm{B}(\mathrm{f})$, the validity of $(\psi, \pi)$ implies that $\psi(r) \bar{s}_{r} \geq 1$, or equivalently that $\psi(r) \geq \bar{\psi}(r)$. For $r<0$, we employ the same reasoning to the solution given by $\bar{s}_{r}=\frac{-f}{r}$ and $\bar{s}_{r^{\prime}}=0$ for $r^{\prime} \neq r$ to obtain $\psi(r) \geq \bar{\psi}(r)$. Finally, we use the solution $\bar{s}_{0}=M>0$, $\bar{s}_{1}=1-f$ and $\bar{s}_{r^{\prime}}=0$ for $r^{\prime} \notin\{0,1\}$ to obtain that $\psi(0) \geq \frac{1-\psi(1)(1-f)}{M}$; taking $M \rightarrow \infty$ gives $\psi(0) \geq 0=\bar{\psi}(0)$.

The second property is proved similarly by considering the feasible solution obtained by setting $\bar{y}_{r}=1, \bar{y}_{1-f-r}=1$ and every other component of $\bar{y}$ to 0 .

Lemma 3. For all $\alpha \in[0,1]$, the function $\left(\bar{\psi}, \bar{\pi}_{\alpha}\right)$ is minimal for $B(f)$.

Proof. Consider $(\psi, \pi)$ such that $\psi \leq \bar{\psi}$ and $\pi \leq \bar{\pi}_{\alpha}$. The first part of Lemma 2 shows that $\psi=\bar{\psi}$. Moreover, elementary calculations show that for all $r, \bar{\pi}_{\alpha}(r)+\bar{\pi}_{\alpha}(1-f-r)=1$. This shows that $\pi=\bar{\pi}_{\alpha}$. Hence the pair $\left(\bar{\psi}, \bar{\pi}_{\alpha}\right)$ is minimal.

\subsection{Extremality}

First we focus on proving Theorem 1 for the case $\alpha \in(0,1]$. It is easy to check that the function $\bar{\pi}_{\alpha}$ is piecewise linear; furthermore, for $\alpha>0$ its breakpoints occur at $\frac{k}{\alpha}$ and $\frac{k}{\alpha}+1-f$ for $k \in \mathbb{Z}$; notice that the first and the second set of breakpoints are respectively the local minima and maxima of $\bar{\pi}_{\alpha}$. Let $\ldots<x_{-2}<x_{-1}<x_{0}=0<x_{1}<x_{2}<\ldots$ be this set of breakpoints; according to this definition, $x_{i}$ is a local minimum for $i$ even. It is easy to check that $\bar{\pi}_{\alpha}$ is quasiperiodic, namely for all $i \in \mathbb{Z}$ and $r \in\left[x_{2 i-1}, x_{2 i+1}\right]$ we have $\bar{\pi}_{\alpha}(r)=\bar{\pi}_{\alpha}\left(x_{2 i}\right)+\bar{\pi}_{\alpha}\left(r-x_{2 i}\right)$.

In order to prove Theorem 11, consider valid functions $\left(\psi_{1}, \pi_{1}\right)$ and $\left(\psi_{2}, \pi_{2}\right)$ satisfying $\bar{\psi}=$ $\frac{\psi_{1}}{2}+\frac{\psi_{2}}{2}$ and $\bar{\pi}_{\alpha}=\frac{\pi_{1}}{2}+\frac{\pi_{2}}{2}$; we show that $\bar{\psi}=\psi_{1}=\psi_{2}$ and $\bar{\pi}_{\alpha}=\pi_{1}=\pi_{2}$.

First notice that Lemma 2 implies $\psi_{1} \geq \bar{\psi}$ and $\psi_{2} \geq \bar{\psi}$; but then since $\frac{\psi_{1}}{2}+\frac{\psi_{2}}{2}=\psi$, it is clear that we must have the equality $\psi_{1}=\psi_{2}=\bar{\psi}$. Therefore, we only need to prove $\pi_{1}=\pi_{2}=\bar{\pi}_{\alpha}$. It is easy to see that $\left(\bar{\psi}, \pi_{1}\right)$ and $\left(\bar{\psi}, \pi_{2}\right)$ are minimal: if there were, say, a valid $\pi_{1}^{\prime} \neq \pi_{1}$ such that $\pi_{1}^{\prime} \leq \pi_{1}$, then $\left(\bar{\psi}, \frac{\pi_{1}^{\prime}}{2}+\frac{\pi_{2}}{2}\right)$ would be a valid function contradicting the minimality of $\left(\bar{\psi}, \bar{\pi}_{\alpha}\right)$.

The proof of Theorem 11 will continue with Claims 1 and 2 below. In order to prove these claims, we need the following technical lemmas about valid functions.

Lemma 4. Consider a minimal valid function $(\psi, \pi)$ for $B(f)$. Then $\pi$ is subadditive, namely for all $r_{1}, r_{2} \in \mathbb{R}, \pi\left(r_{1}+r_{2}\right) \leq \pi\left(r_{1}\right)+\pi\left(r_{2}\right)$. 
Proof. We first prove $\pi(0) \geq 0$. For a positive integer $M$, consider the following feasible solution for $\mathrm{B}(\mathrm{f}): \bar{x}=1, \bar{s}_{1}=1-f, \bar{s}_{r}=0$ for $r \neq 1, \bar{y}_{0}=M$, and $\bar{y}_{r}=0$ for $r \neq 0$. The validity of $(\psi, \pi)$ implies that $\pi(0) \geq \frac{1-\psi(1)(1-f)}{M}$. Taking $M \rightarrow \infty$ gives $\pi(0) \geq 0$.

Now take $r_{1}, r_{2} \in \mathbb{R}$. We want to show $\pi\left(r_{1}+r_{2}\right) \leq \pi\left(r_{1}\right)+\pi\left(r_{2}\right)$. This holds when $r_{1}=0$ or $r_{2}=0$, since $\pi(0) \geq 0$. Assume now that $r_{1}, r_{2} \neq 0$. Define the function $\pi^{\prime}$ as follows:

$$
\pi^{\prime}(r)= \begin{cases}\pi\left(r_{1}\right)+\pi\left(r_{2}\right) & \text { if } r=r_{1}+r_{2} \\ \pi(r) & \text { if } r \neq r_{1}+r_{2} .\end{cases}
$$

We show that $\left(\psi, \pi^{\prime}\right)$ is valid. Consider any $(\bar{x}, \bar{s}, \bar{y}) \in B(f)$. Define $\tilde{y}$ as follows:

$$
\tilde{y}_{r}= \begin{cases}\bar{y}_{r_{1}}+\bar{y}_{r_{1}+r_{2}} & \text { if } r=r_{1} \\ \bar{y}_{r_{2}}+\bar{y}_{r_{1}+r_{2}} & \text { if } r=r_{2} \\ 0 & \text { if } r=r_{1}+r_{2} \\ \bar{y}_{r} & \text { otherwise. }\end{cases}
$$

Using the definitions of $\pi^{\prime}$ and $\tilde{y}$, it follows that

$$
\sum_{r} \pi(r) \tilde{y}_{r}=\sum_{r} \pi^{\prime}(r) \bar{y}_{r}
$$

Furthermore we have $\sum_{r} r \tilde{y}_{r}=\sum_{r} r \bar{y}_{r}$, which implies that $(\bar{x}, \bar{s}, \tilde{y})$ belongs to $B(f)$.

Since $(\psi, \pi)$ is valid, $\sum_{r} \psi(r) \bar{s}_{r}+\sum_{r} \pi(r) \tilde{y}_{r} \geq 1$, and therefore $\sum_{r} \psi(r) \bar{s}_{r}+\sum_{r} \pi^{\prime}(r) \bar{y}_{r} \geq 1$. Thus, $\left(\psi, \pi^{\prime}\right)$ is valid. Since $\pi$ is minimal, we get $\pi\left(r_{1}+r_{2}\right) \leq \pi^{\prime}\left(r_{1}+r_{2}\right)=\pi\left(r_{1}\right)+\pi\left(r_{2}\right)$.

Lemma 5. If $(\psi, \pi)$ is a valid function for $B(f)$, then $(\psi, \min \{\psi, \pi\})$ is also valid for $B(f)$.

Proof. Consider any $(\bar{x}, \bar{s}, \bar{y})$ in $B(f)$. Define $(\bar{x}, \tilde{s}, \tilde{y})$ as follows:

$$
\begin{gathered}
\tilde{s}_{r}= \begin{cases}\bar{s}_{r} & \text { if } \psi(r) \geq \pi(r) \\
\bar{s}_{r}+\bar{y}_{r} & \text { if } \psi(r)<\pi(r)\end{cases} \\
\tilde{y}_{r}= \begin{cases}\bar{y}_{r} & \text { if } \psi(r) \geq \pi(r) \\
0 & \text { if } \psi(r)<\pi(r) .\end{cases}
\end{gathered}
$$

Since $(\bar{x}, \tilde{s}, \tilde{y})$ belongs to $B(f)$, we have $\sum_{r} \psi(r) \tilde{s}_{r}+\sum_{r} \pi(r) \tilde{y}_{r} \geq 1$. Using the definition of $\tilde{s}$ and $\tilde{y}$, we get $\sum_{r} \psi(r) \bar{s}_{r}+\sum_{r} \min \{\psi(r), \pi(r)\} \bar{y}_{r} \geq 1$, therefore $(\psi, \min \{\psi, \pi\})$ is valid for $B(f)$.

Claim 1. For $r \in\left[x_{-1}, x_{1}\right], \pi_{1}(r)=\pi_{2}(r)=\bar{\psi}(r)=\bar{\pi}_{\alpha}(r)$.

Proof. Fix $j \in\{1,2\}$. Since $\left(\bar{\psi}, \pi_{j}\right)$ is valid for $B(f)$, Lemma 5 implies that $\left(\bar{\psi}, \min \left\{\bar{\psi}, \pi_{j}\right\}\right)$ is also valid for $B(f)$. The minimality of $\left(\bar{\psi}, \pi_{j}\right)$ then implies that $\pi_{j} \leq \min \left\{\bar{\psi}, \pi_{j}\right\} \leq \bar{\psi}$.

However, notice that for $r \in\left[x_{-1}, x_{1}\right], \bar{\psi}(r)=\bar{\pi}_{\alpha}(r)$. As before, for $r \in\left[x_{-1}, x_{1}\right]$ the fact that $\bar{\psi}(r)=\pi_{1}(r)+\pi_{2}(r)$ together with the previous paragraph implies $\pi_{1}(r)=\pi_{2}(r)=\bar{\psi}(r)$. The result then follows.

Claim 2. For $r \in\left[x_{2 i-1}, x_{2 i+1}\right]$ and $j=1,2$, we have

$$
\pi_{j}(r)-\pi_{j}\left(x_{2 i}\right)=\bar{\pi}_{\alpha}(r)-\bar{\pi}_{\alpha}\left(x_{2 i}\right) .
$$

Proof. Since $\pi_{j}$ is minimal, Lemma 4 gives that $\pi_{j}(r) \leq \pi_{j}\left(x_{2 i}\right)+\pi_{j}\left(r-x_{2 i}\right)$. But since $r-x_{2 i} \in\left[x_{-1}, x_{1}\right]$, it follows from Claim 1 and the quasiperiodicity of $\bar{\pi}_{\alpha}$ that $\pi_{j}\left(r-x_{2 i}\right)=$ $\bar{\pi}_{\alpha}\left(r-x_{2 i}\right)=\bar{\pi}_{\alpha}(r)-\bar{\pi}_{\alpha}\left(x_{2 i}\right)$; this gives that $\pi_{j}(r)-\pi_{j}\left(x_{2 i}\right) \leq \bar{\pi}_{\alpha}(r)-\bar{\pi}_{\alpha}\left(x_{2 i}\right)$ for $j=1,2$. Adding these inequalities for $j=1,2$, dividing by 2 and using the fact that $\bar{\pi}_{\alpha}=\frac{\pi_{1}}{2}+\frac{\pi_{2}}{2}$, we again obtain that we must have the equality $\pi_{j}(r)-\pi_{j}\left(x_{2 i}\right)=\bar{\pi}_{\alpha}(r)-\bar{\pi}_{\alpha}\left(x_{2 i}\right)$ for $j=1,2$. This proves the claim. 
Now take $i \in \mathbb{N}$, and $r \in\left[x_{2 i-1}, x_{2 i+1}\right]$; we will show that $\pi_{1}(r)=\pi_{2}(r)=\bar{\pi}_{\alpha}(r)$. For that, simply write $\pi_{j}(r)=\left(\pi_{j}(r)-\pi_{j}\left(x_{2 i}\right)\right)+\sum_{k=1}^{2 i}\left(\pi_{j}\left(x_{k}\right)-\pi_{j}\left(x_{k-1}\right)\right)+\pi_{j}\left(x_{0}\right)$. Applying Claim 2 to each parenthesized expression and Claim 1 to the last term, we obtain that $\pi_{j}(r)=\bar{\pi}_{\alpha}(r)$, giving the desired result. The case $i \in-\mathbb{N}$ can be handled analogously, which then proves Theorem 1 when $\alpha \in(0,1]$.

The case $\alpha=0$ needs to be handled separately. As before, we still have $\psi_{1}=\psi_{2}=\bar{\psi}$. Moreover, as in Claim 1, the fact that $\bar{\pi}_{0}(r)=\bar{\psi}(r)$ for all $r \leq 1-f$ implies that $\pi_{1}(r)=$ $\pi_{2}(r)=\bar{\pi}_{0}(r)$ for all $r \leq 1-f$. For $r>1-f$, we claim that we have an equality analogous to Claim 2, namely that $\pi_{j}(r)-\pi_{j}(1-f)=\bar{\pi}_{0}(r)-\bar{\pi}_{0}(1-f)$ for $j=1,2$. To see this, first notice that for $r>1-f$ we have $\bar{\pi}_{0}(r)=\bar{\pi}_{0}(1-f)-\bar{\pi}_{0}(1-f-r)$. Then using the subadditivity of $\pi_{j}$ and the fact that $1-f-r \leq 1-f$, we get $\pi_{j}(1-f) \leq \pi_{j}(1-f-r)+\pi_{j}(r)=\bar{\pi}_{0}(1-f)-\bar{\pi}_{0}(r)+\pi_{j}(r)$, and the claim follows as in Claim 2. Since $\pi_{j}(1-f)=\bar{\pi}_{0}(1-f)$, this equation gives that $\pi_{j}(r)=\bar{\pi}_{0}(r)$ for all $r>1-f$ and $j=1,2$. This concludes the proof of Theorem 1 ,

\subsection{Split cuts}

Let $L P$ denote the linear relaxation of formulation $I P$, i.e. it is obtained from $I P$ by replacing the integrality conditions $x \in\{-\infty, \ldots, 0,1\}, y_{r} \in \mathbb{Z}_{+}$by $x \leq 1, y_{r} \geq 0$. We say that an inequality $\sum_{r} \psi(r) s_{r}+\sum_{r} \pi(r) y_{r} \geq 1$ is a split inequality if it is satisfied by all $(x, s, y)$ in $L P \cap\left(\left\{a x+\sum_{r} b_{r} y_{r} \leq c\right\} \cup\left\{a x+\sum_{r} b_{r} y_{r} \geq c+1\right\}\right)$, for some $a, c \in \mathbb{Z}, b_{r} \in \mathbb{Z}$ for all $r \in \mathbb{R}$.

Note that split disjunctions $\left\{a x+\sum_{r} b_{r} y_{r} \leq c\right\} \cup\left\{a x+\sum_{r} b_{r} y_{r} \geq c+1\right\}$ are valid for $I P$ even when we relax the upper bound on $x$, namely when we simply take $x$ to be an integer. In other words, split inequalities do not use the upper bound information on $x$. This is in contrast with the wedge inequalities (1), which use the upper bound on $x$, being derived from the disjunction $\left\{(x, z, s, y):(x, z) \notin \operatorname{int} K_{\alpha}\right\}$ (recall Figure 1). It is therefore somewhat surprising that every wedge inequality is dominated by a split inequality.

We will prove this in Theorem 2. Intuitively, $L P$ contains the constraint $x \leq 1$ and, restricting our attention to the $(x, z)$-space, we see that $K_{\alpha} \cap\{x \leq 1\}$ is contained in a split intersected with $\{x \leq 1\}$ (see Figure 4). The gist of the proof of Theorem 2 is to show that this inclusion can be translated into domination of the corresponding inequalities.

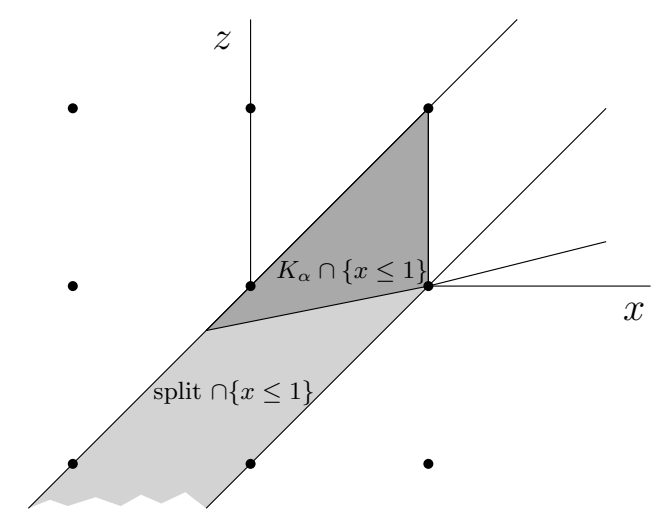

Figure 4: $K_{\alpha} \cap\{x \leq 1\}$ is contained in a split.

Theorem 2. For all $\alpha \in(0,1]$ and all $\ell: \mathbb{R} \rightarrow \mathbb{Z}$, the wedge inequality (10) is a split inequality.

Proof. To prove the theorem, we show that the wedge inequality (10) is satisfied by all $(x, s, y)$ in $L P \cap\left(\left\{x \leq \sum_{r} \ell(r) y_{r}\right\} \cup\left\{x-1 \geq \sum_{r} \ell(r) y_{r}\right\}\right)$.

Let $\beta=\frac{\alpha f}{1-\alpha(1-f)}$. Notice that $0<\beta \leq 1$ since $0<\alpha \leq 1$. 
Claim 1 If $(\bar{x}, \bar{s}, \bar{y}) \in L P \cap\left(\left\{x \leq \sum_{r} \ell(r) y_{r}\right\} \cup\left\{x-1 \geq \sum_{r} \ell(r) y_{r}\right\}\right)$, then $(\bar{x}, \bar{s}, \bar{y}) \in L P \cap(\{x \leq$ $\left.\left.\sum_{r} \ell(r) y_{r}\right\} \cup\left\{\beta(x-1) \geq \sum_{r} \ell(r) y_{r}\right\}\right)$.

Proof. If $(\bar{x}, \bar{s}, \bar{y}) \in L P \cap\left\{x \leq \sum_{r} \ell(r) y_{r}\right\}$, then the claim holds. Now suppose $(\bar{x}, \bar{s}, \bar{y}) \in$ $L P \cap\left\{x-1 \geq \sum_{r} \ell(r) y_{r}\right\}$. Since $0<\beta \leq 1$, and $x \leq 1$ is a constraint of $L P$, we have $(1-\beta)(\bar{x}-1) \leq 0$. Hence, $\sum_{r} \ell(r) \bar{y}_{r} \leq(\bar{x}-1)-(1-\beta)(\bar{x}-1)=\beta(\bar{x}-1)$ and we are done.

To finish the proof of the theorem, it remains to show that (11) is valid for $L P \cap(\{x \leq$ $\left.\left.\sum_{r} \ell(r) y_{r}\right\} \cup\left\{\beta(x-1) \geq \sum_{r} \ell(r) y_{r}\right\}\right)$. To see this, consider the function $\psi: \mathbb{R}^{2} \rightarrow \mathbb{R}$ defined as $\psi(r, \ell)=\max \left\{a^{1} \cdot(r, \ell), a^{2} \cdot(r, \ell)\right\}$. It is easy to verify that $\psi$ is convex, and positively homogeneous, and thus it is subadditive, i.e., $\psi(r, \ell)+\psi\left(r^{\prime}, \ell^{\prime}\right) \geq \psi\left(r+r^{\prime}, \ell+\ell^{\prime}\right)$. Moreover, $\psi(x-f, z)=1$ on the boundary of the set $K_{\alpha}$. By convexity of $\psi$ and $K_{\alpha}$, we get that $\psi(x-f, z) \geq 1$ if $(x, z) \notin \operatorname{int} K_{\alpha}$, i.e. $x \leq z$ or $\beta(x-1) \geq z$. Using the equations for $(x, z)$ in the definition of $I P(\ell)$, we derive the following valid inequality for all $(s, y)$ such that $(x, z)$ satisfies $x \leq z$ or $\beta(x-1) \geq z$ :

$$
1 \leq \psi(x-f, z)=\psi\left(\sum_{r}(r, 0) s_{r}+\sum_{r}(r, \ell(r)) y_{r}\right) \leq \sum_{r} \psi(r, 0) s_{r}+\sum_{r} \psi(r, \ell(r)) y_{r} .
$$

Substituting $\sum_{r} \ell(r) y_{r}$ for $z$ into the inequalities $x \leq z$ or $\beta(x-1) \geq z$ gives the desired result.

We obtain as a corollary a result for the cuts $\left(\bar{\psi}, \bar{\pi}_{\alpha}\right)$, including the case $\alpha=0$.

Corollary 1. For all $\alpha \in[0,1]$, the inequality generated by $\left(\bar{\psi}, \bar{\pi}_{\alpha}\right)$ is a split inequality.

Proof. It suffices to prove for $\alpha=0$. Consider any feasible solution $(x, s, y)$ in $L P \cap(\{(x, s, y)$ : $\left.\left.x \leq \sum_{r>1-f} y_{r}\right\} \cup\left\{(x, s, y): x \geq 1+\sum_{r>1-f} y_{r}\right\}\right)$. Since $s$ and $y$ have finite support, Lemma 1 implies that there exists $\alpha_{0}>0$ such that: (i) $\ell_{\alpha_{0}}(r)$ equals 0 if $r \leq 1-f$ and equals 1 if $r>1-f$; (ii) $\bar{\pi}_{\alpha_{0}}(r)=\bar{\pi}_{0}(r)$ for all $r$ such that $s_{r} \neq 0$ or $y_{r} \neq 0$. Property (i) and the proof of Theorem 2 give that $(x, s, y)$ satisfies the inequality defined by $\left(\bar{\psi}, \bar{\pi}_{\alpha_{0}}\right)$. Property (ii) then shows that $(x, s, y)$ also satisfies the inequality defined by $\left(\bar{\psi}, \bar{\pi}_{0}\right)$. This concludes the proof.

\section{Computations}

We performed computational tests to assess the practical consequences of Theorem 2 of Section 4.3. We have selected 63 instances from MIPLIB 2010 with binary and continuous variables only. We have generated wedge cuts $\left(\bar{\psi}, \bar{\pi}_{\alpha}\right)$ with $\alpha \in\{0,0.5,0.8,0.85,0.9,0.95,1\}$ for the most fractional binary variables in the optimal basis of the LP relaxation. For the experiments we have used the LP solver of FICO Xpress with no preprocessing at all. For each instance, and each fixed $\alpha<1$, we generated one round of $50+50$ wedge cuts for strengthening the LP relaxation (50 for each one of the two orientations of the cone $K_{\alpha}$, cf. Section 3). For $\alpha=1$, when wedge cuts are equivalent to GMI cuts by Proposition 2, we have generated one round of 50 cuts. Hence, for each instance we have a total of 7 runs of our cutting plane algorithm, and in each run we have added cuts with a single fixed $\alpha$ parameter only. Let $L B(I)$ and $L B_{\alpha}^{+}(I)$ denote the optimum value of the LP relaxation, and the value after adding one round of cuts of parameter $\alpha$, respectively, on instance $I$. For each instance $I$, and parameter $\alpha$, we have computed the quantity $A_{\alpha}(I)=\left(L B_{\alpha}^{+}(I)-L B(I)\right) /|L B(I)+1|$, where the denominator is perturbed by 1 to handle those cases with $L B(I)=0$. Let $\bar{A}_{\alpha}$ denote the average of the $A_{\alpha}(I)$ values over the 63 instances for each $\alpha$. Table 1 depicts the averages.

Observe that as $\alpha$ tends to 1 (i.e., the cuts approach GMI cuts), the improvement over the LP optimum strictly increases. Our findings complement those of Balas and Qualizza [2] which 


\begin{tabular}{|l|cccccc|c|}
\hline$\alpha$ & 0 & 0.5 & 0.8 & 0.85 & 0.9 & 0.95 & 1 (GMI) \\
\hline $\bar{A}_{\alpha}$ & 0.1305 & 0.131 & 0.136 & 0.139 & 0.141 & 0.144 & 0.146 \\
\hline
\end{tabular}

Table 1: Average improvement over the LP optimum for increasing $\alpha$ values.

showed that in practice lopsided cuts do not improve much over GMI cuts from the tableau, although they are occasionally stronger. On average, they tend to be weaker. What these results indicate is that it does not pay to use bounds on the basic variables when generating GMI cuts from tableau rows. Instead it is preferable to generate the standard GMI cut and to simply use the bounds directly in the formulation. This seems counterintuitive but it is in agreement with the proof of Theorem 2 ,

\section{References}

[1] Egon Balas and Robert B. Jeroslow, Strengthening Cuts for Mixed Integer Programs, European Journal of Operations Research 4 (1980) 224-234.

[2] Egon Balas and Andrea Qualizza, Monoidal Cut Strengthening Revisited, Discrete Optimization 9 (2012) 40-49.

[3] Amitabh Basu, Michele Conforti, Gerard Cornuejols and Giacomo Zambelli, Minimal Inequalities for an Infinite Relaxation of Integer Programs, SIAM Journal on Discrete Mathematics 24 (2010) 158-168.

[4] Michele Conforti, Gerard Cornuejols and Giacomo Zambelli, A Geometric Perspective on Lifting, Operations Research 59 (2011) 569-577.

[5] Ralph E. Gomory and Ellis L. Johnson, Some Continuous Functions Related to Corner Polyhedra I, Mathematical Programming 3 (1972) 23-85. 